\title{
The Distance 4-Sector of Two Points is Unique
}

\author{
Robert Fraser ${ }^{1}$, Meng $\mathrm{He}^{2}$, Akitoshi Kawamura ${ }^{3}$, Alejandro López-Ortiz ${ }^{4}$, \\ J. Ian Munro ${ }^{4}$, and Patrick K. Nicholson ${ }^{5}$ \\ 1 Department of Computer Science, University of Manitoba, Winnipeg, Canada, \\ fraser@cs. umanitoba.ca \\ 2 Faculty of Computer Science, Dalhousie University, Halifax, Canada, \\ mhe@cs.dal.ca \\ 3 Department of Computer Science, University of Tokyo, Tokyo, Japan, \\ kawamura@is.s.u-tokyo.ac.jp \\ ${ }^{4}$ Cheriton School of Computer Science, University of Waterloo, Waterloo, Canada, \\ \{alopez-o, imunro\}@cs. uwaterloo.ca \\ 5 Max-Planck-Institut für Informatik, Saarbrücken, Germany, \\ pnichols@mpi-inf.mpg. de
}

\begin{abstract}
The (distance) $k$-sector is a generalization of the concept of bisectors proposed by Asano, Matoušek and Tokuyama. We prove the uniqueness of the 4-sector of two points in the Euclidean plane. Despite the simplicity of the unique 4-sector (which consists of a line and two parabolas), our proof is quite non-trivial. We begin by establishing uniqueness in a small region of the plane, which we show may be perpetually expanded afterward.
\end{abstract}

Keywords: distance $k$-sector, Tarski fixed point, uniqueness

\section{Introduction}

The bisector of two nonempty sets $X$ and $Y$ in $\mathbb{R}^{2}$ is defined as

$$
\operatorname{bisect}(X, Y)=\left\{z \in \mathbb{R}^{2}: \operatorname{dist}(z, X)=\operatorname{dist}(z, Y)\right\},
$$

where $\operatorname{dist}(z, X)=\inf _{x \in X} \operatorname{dist}(z, x)$ denotes the Euclidean distance of $z$ from a set $X$. For any integer $k \geq 2$, a distance $k$-sector (or simply $k$-sector) of distinct points $p, q \in \mathbb{R}^{2}$ is a $(k-1)$-tuple $\left(C_{1}, \ldots, C_{k-1}\right)$ of nonempty subsets of $\mathbb{R}^{2}$ such that

$$
C_{i}=\operatorname{bisect}\left(C_{i-1}, C_{i+1}\right), \quad i=1, \ldots, k-1,
$$

where $C_{0}=\{p\}$ and $C_{k}=\{q\}$.

For example, there is a 4 -sector of two points that consists of a line and two parabolas (Fig. 1). We will prove that this is the only one:

Theorem 1. The 4-sector between two points in the Euclidean plane is unique. 


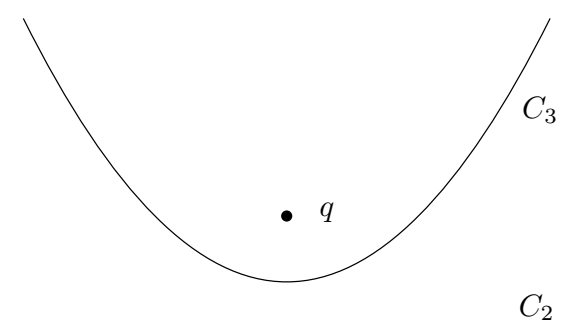

$C_{2}$

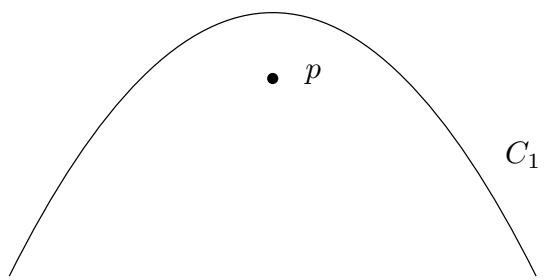

Fig. 1. A 4-sector $\left(C_{1}, C_{2}, C_{3}\right)$ of $p$ and $q$ (whose uniqueness we will prove). If $p=$ $(0,-1)$ and $q=(0,1)$, these curves are the graphs of $y= \pm\left(x^{2}+1\right) / 2$ and $y=0$.

The notion of distance $k$-sectors was introduced by Asano and Tokuyama [4] in 2004, motivated by a question about circuit board design. Asano, Matoušek and Tokuyama [3] showed the existence and uniqueness of the 3 -sector (trisector). Despite the simple definition, $k$-sectors (with the exception of $k=2,4$ ) do not seem to be easy to construct (note that bisecting between the curves in Fig. 1 does not give an 8 -sector). In particular, the 3 -sector is not algebraic, as conjectured in [3] and recently proved by Monterde and Ongay [8].

Although the original proof in [3] of the existence and uniqueness of the 3sector was rather involved, it turned out later that $k$-sectors exist in a fairly general setting and for a relatively simple reason: Reem and Reich [9] used the Tarski fixed point theorem to prove the existence of a closely related object called double zone diagrams. Applying this idea, Imai et al. [6] proved the existence of a $k$-sector for any $k$ on any sets $P, Q$ (instead of $\{p\},\{q\}$ ), and for a general class of metric spaces. The existence proofs sometimes suggest an algorithm to compute (draw on a screen approximately) $k$-sectors, but its efficiency is unclear. Some issues in computing $k$-sectors and double zone diagrams are discussed in $[6,10]$ (of course, such issues are trivial in our setting of 4-secting two points, once we show that the only 4-sector is the one explicitly given in Fig. 1).

Uniqueness is harder to prove. The uniqueness of the 3 -sector was extended to the case where one of $P, Q$ is a line segment instead of a point [5], and then to the general case where $P, Q$ can be any disjoint nonempty closed sets [7] (in fact, they proved the uniqueness of the zone diagram [2], a generalization of 3-sectors where we start with many sets instead of just two sets $P, Q)$. Unlike existence, uniqueness relies on the properties of the Euclidean norm, and indeed fails for, say, the $l_{1}$ norm $[1,7]$. It remains open [6, Conjecture] whether $k$-sectors, for 
$k \geq 4$, are unique even in the Euclidean plane. Theorem 1 answers this for $k=4$ (and points $p, q$ ).

Proof idea. Roughly speaking, the central part of our proof (Lemma 3) is based on the following ideas. Suppose that there are two different $k$-sectors $\left(C_{i}\right)_{i}$ and $\left(\hat{C}_{i}\right)_{i}$ (here and in the sequel, the subscript $i$ always ranges over $1, \ldots, k-1$, so that $\left(C_{i}\right)_{i}$ means $\left.\left(C_{1}, \ldots, C_{k-1}\right)\right)$. Then there is a gap somewhere between the curves $C_{i}$ and $\hat{C}_{i}$. Since they both satisfy equation (2), we must have another gap somewhere between $C_{i-1}$ and $\hat{C}_{i-1}$ or between $C_{i+1}$ and $\hat{C}_{i+1}$, which is not too small compared to the original gap. From some observations about the size and location of the new gap, we derive contradiction by arguing that this process of finding a new gap cannot go on forever because it causes the gap to grow too big to fit where it must be. This rough intuition is common to the proof of 3 -sector uniqueness in [7], but there is a lot of room for creativity as to how we define the size of the gap between two curves at a point. The proof in [7] used a clever way to measure the gap under which the gap always grows bigger, but this measure of the gaps only makes sense for 3 -sectors. We measure the gap much more simply, by the difference between the y-coordinates of the two curves at a common $\mathrm{x}$-coordinate. The downside is that under this measure, the gap gets bigger only when the involved parts of the curves lie in certain configuration. This necessitates some detailed argument that certain part of 4-sector indeed has this configuration (Lemma 6) and that the uniqueness of this part of the 4 -sector can then be extended gradually to other parts (Lemmas 4 and 5).

\section{Preliminaries: Gradations}

In the definition of $k$-sectors above, the components $C_{i}$ are sets satisfying certain equations, and we did not even say that they are curves. It is not entirely obvious, although it is true, that each of them divides the plane into two regions, one containing $p$ and the other containing $q$. Imai et al. [6] made this claim precise (as Lemma 1 below) by introducing $k$-gradations. We briefly review their definition, as we also find $k$-gradations easier to reason with than $k$-sectors.

For nonempty $X, Y \subseteq \mathbb{R}^{2}$, we define the dominance region of $X$ over $Y$ by

$$
\operatorname{dom}(X, Y)=\left\{z \in \mathbb{R}^{2}: \operatorname{dist}(z, X) \leq \operatorname{dist}(z, Y)\right\} .
$$

It is not hard to see [6, Lemma 6] that, if $X$ and $Y$ are disjoint closed sets, $\operatorname{bisect}(X, Y)$ is the boundary of $\operatorname{dom}(X, Y)$. A $k$-gradation between points $p$, $q \in \mathbb{R}^{2}$ is a $(k-1)$-tuple $\left(R_{i}, S_{i}\right)_{i}$ of pairs of subsets of $\mathbb{R}^{2}$ satisfying

$$
R_{i}=\operatorname{dom}\left(R_{i-1}, S_{i+1}\right), \quad S_{i}=\operatorname{dom}\left(S_{i+1}, R_{i-1}\right), \quad i=1, \ldots, k-1,
$$

where $R_{0}=\{p\}$ and $S_{k}=\{q\}$. It is easy to see that this implies $R_{i} \cup S_{i}=\mathbb{R}^{2}$, $\{p\}=R_{0} \subseteq R_{1} \subseteq \cdots \subseteq R_{k-1}$ and $S_{1} \supseteq S_{2} \supseteq \cdots \supseteq S_{k}=\{q\}$.

By the following lemma (a special case of [6, Proposition 2]), a $k$-sector $\left(C_{i}\right)_{i}$ can be identified with a $k$-gradation $\left(R_{i}, S_{i}\right)_{i}$ : 
Lemma $1([6]) . A(k-1)$-tuple $\left(C_{i}\right)_{i}$ of sets is a $k$-sector of points $p, q \in \mathbb{R}^{2}$ if and only if

$$
C_{i}=R_{i} \cap S_{i}, \quad i=1, \ldots, k-1
$$

for some $k$-gradation $\left(R_{i}, S_{i}\right)_{i}$ between $p, q$.

Imai et al. [6, Proposition 1] established the existence of a $k$-sector by proving the existence of a $k$-gradation:

Lemma 2 ([6]). There exists a k-gradation between two distinct points in $\mathbb{R}^{2}$. In fact, there are the greatest and the least $k$-gradations under the order defined by: $\left(R_{i}, S_{i}\right)_{i} \leq\left(R_{i}^{\prime}, S_{i}^{\prime}\right)_{i}$ if $R_{i} \subseteq R_{i}^{\prime}$ and $S_{i} \supseteq S_{i}^{\prime}$ for all $i=1, \ldots, k-1$.

In what follows, we will primarily deal with $k$-gradations as opposed to $k$ sectors, as their use allows the proofs to be simpler and cleaner.

\section{Proof of Theorem 1}

For the rest of the paper, we fix $p=(0,-1)$ and $q=(0,+1)$ and consider the 4 -sectors and 4-gradations between them. As explained above (Lemma 1), each 4 -sector $\left(C_{i}\right)_{i}$ corresponds to a 4 -gradation $\left(R_{i}, S_{i}\right)_{i}$. We will prove that this 4-gradation agrees with $\left(\hat{R}_{i}, \hat{S}_{i}\right)_{i}$, the trivial 4 -gradation corresponding to the trivial 4-sector $\left(\hat{C}_{i}\right)_{i}$ described in Fig. 1.

Define the (closed) regions $I_{\beta}$ and $J_{\beta}$, for $\beta>0$, as follows (Fig. 2), using the trivial 4 -sector $\left(\hat{C}_{i}\right)_{i}$. Consider the normal lines to the parabolas $\hat{C}_{1}$ and $\hat{C}_{3}$ at $x= \pm \beta$. The closed, finite region of the plane (containing the origin) defined by these lines is $I_{\beta}$. The region $J_{\beta}$ contains $I_{\beta}$, as well as all points lying above the upper envelope of the normal lines to $\hat{C}_{1}$ and all points lying below the lower envelope of the normal lines to $\hat{C}_{3}$. Calculation shows that the four vertices of $I_{\beta}$ are $\left( \pm\left(3+\beta^{2}\right) \beta / 2,0\right)$ and $\left(0, \pm\left(3+\beta^{2}\right) / 2\right)$.

We prove Theorem 1 using the following three lemmas. Note that the number 0.5774 in the lemmas is slightly greater than $1 / \sqrt{3}$, so that the boundary halflines of $J_{0.5774}$ make angle slightly less than $\pi / 3$ with the x-axis.

Lemma 3. Let $\left(R_{i}, S_{i}\right)_{i}$ be a 4-gradation. Then $R_{2}$ and $S_{2}$ agree with the trivial 4-gradation on $J^{\prime}:=J_{0.5774} \cap(\mathbb{R} \times[-4,4])$, i.e., $R_{2} \cap J^{\prime}=\hat{R}_{2} \cap J^{\prime}$ and $S_{2} \cap J^{\prime}=$ $\hat{S}_{2} \cap J^{\prime}$.

Lemma 4. Let $\beta \geq 0.5774$. Let $\left(R_{i}, S_{i}\right)_{i}$ be a 4-gradation. Suppose that $R_{2}$ and $S_{2}$ agree with the trivial 4-gradation on $J_{\beta}$, i.e., $R_{2} \cap J_{\beta}=\hat{R}_{2} \cap J_{\beta}$ and $S_{2} \cap J_{\beta}=\hat{S}_{2} \cap J_{\beta}$. Then $R_{1}, S_{1}, R_{3}, S_{3}$ agree with the trivial 4-gradation on a region $I_{\beta^{\prime}}$, for some $\beta^{\prime} \geq 1.00001 \beta$. Moreover, for $\beta=0.5774$, this holds with $J_{\beta}$ replaced by the $J^{\prime}$ defined in Lemma 3.

Lemma 5. Let $\beta>0$. Let $\left(R_{i}, S_{i}\right)_{i}$ be a 4-gradation. Suppose that $R_{1}, S_{1}$, $R_{3}, S_{3}$ agree with the trivial 4-gradation on $I_{\beta}$, i.e., $R_{i} \cap I_{\beta}=\hat{R}_{i} \cap I_{\beta}$ and $S_{i} \cap I_{\beta}=\hat{S}_{i} \cap I_{\beta}$ for each $i=1,3$. Then $R_{2}, S_{2}$ agree with the trivial 4-gradation on $J_{\beta}$, i.e., $R_{2} \cap J_{\beta}=\hat{R}_{2} \cap J_{\beta}$ and $S_{2} \cap J_{\beta}=\hat{S}_{2} \cap J_{\beta}$. 


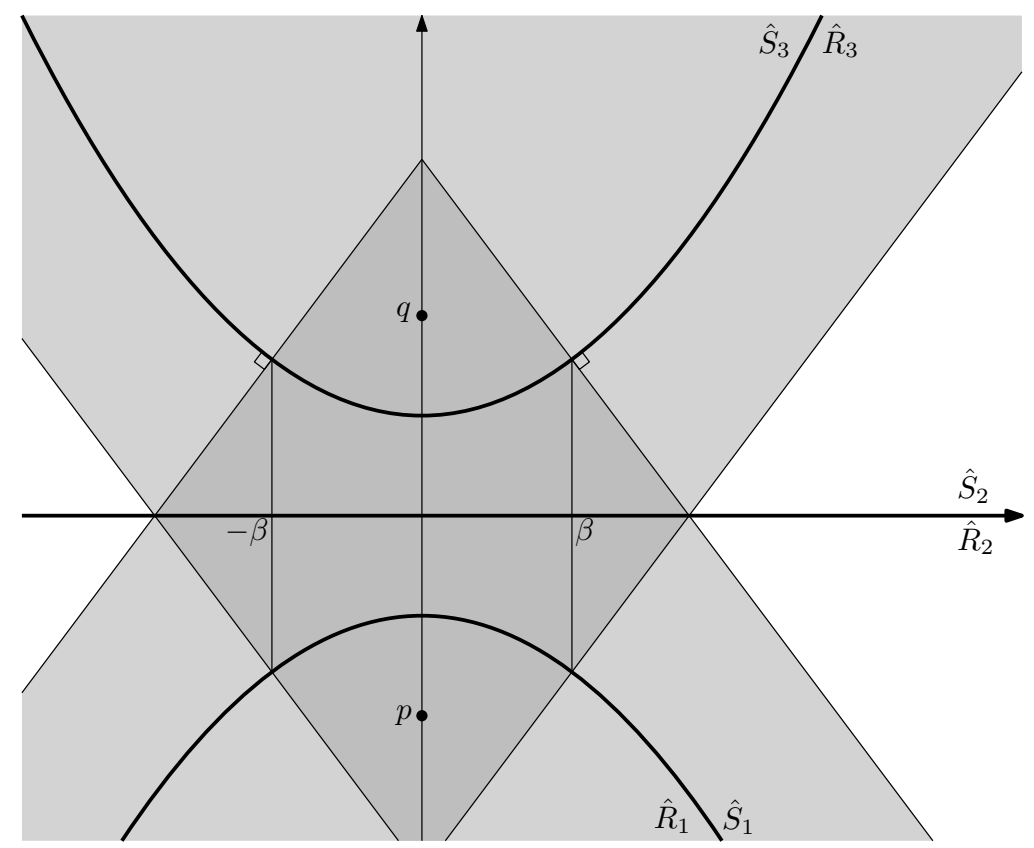

Fig. 2. Closed regions $I_{\beta}$ (dark grey) and $J_{\beta}$ (light grey plus dark grey), defined from the trivial 4-gradation $\left(\hat{R}_{i}, \hat{S}_{i}\right)_{i}$.

Lemma 3 is the central part of our proof: it says that the middle component $\left(R_{2}, S_{2}\right)$ of the 4-gradation is uniquely determined (and agrees with the trivial one) up to a certain point (i.e., in the region $\left.J^{\prime}\right)$. Lemma 4 says that if $\left(R_{2}, S_{2}\right)$ is uniquely determined up to some point, then it uniquely determines $\left(R_{1}, S_{1}\right)$ and $\left(R_{3}, S_{3}\right)$ up to some point. Lemma 5 works the other way, stating that a certain part of $\left(R_{2}, S_{2}\right)$ is determined by certain parts of $\left(R_{1}, S_{1}\right)$ and $\left(R_{3}, S_{3}\right)$.

To prove Theorem 1, we use Lemma 3 followed by the last sentence (the "moreover" part) of Lemma 4, and then apply Lemmas 5 and 4 alternately, extending step by step the region on which the considered 4-gradation agrees with the trivial one. This proves the uniqueness of the 4-gradation, and thus, by Lemma 1, of the 4-sector.

The proofs of the lemmas require a number of additional properties, and so their presentation is delayed to Sections 3.2 and 3.3 .

\subsection{Properties of a 4-Gradation}

Before proving the lemmas, we need to get some rough estimates about what a 4-gradation must look like. Let $\mathrm{B}(w, r)$ (resp. $\left.\mathrm{B}^{\circ}(w, r)\right)$ denote the closed (resp. open) ball with centre $w$ and radius $r$.

Lemma 6. Let $\left(R_{i}, S_{i}\right)_{i}$ be a 4-gradation. Then 
1. $R_{1}$ and $S_{3}$ are convex.

2. $\operatorname{dist}\left(R_{i}, S_{i+1}\right) \geq 1 / 2$ for each $i=0,1,2,3$.

3. $\mathrm{B}(p, i / 2) \subseteq R_{i}$ and $\mathrm{B}(q, i / 2) \subseteq S_{4-i}$ for each $i=0,1,2,3,4$.

4. $R_{1} \subseteq \mathbb{R} \times(-\infty,-1 / 2]$ and $S_{3} \subseteq \mathbb{R} \times[1 / 2, \infty)$.

5. Let $w=(a, b)$ be a point with $a \geq 0$ and $b \leq 0$. Let $v=\left(a^{\prime}, b^{\prime}\right)$ be a closest point to $w$ in $S_{3}$. Then,

(a) $0 \leq a^{\prime} \leq a$.

(b) If $|a| \leq 1.16$, then $b^{\prime} \in[1 / 2,0.82]$.

Likewise for $S_{3}$ and $b \leq 0$ replaced by $R_{1}$ and $b \geq 0$, respectively.

6. For each $t>0$, let $L_{t}^{ \pm}=\left\{(x, y) \in \mathbb{R}^{2}: \pm y \leq x^{2} / t\right\}$, respectively. Then $R_{2} \subseteq L_{4}^{+}$and $S_{2} \subseteq L_{4}^{-}$.

Proof. 1. $R_{1}=\operatorname{dom}\left(p, S_{2}\right)=\bigcap_{s \in S_{2}} \operatorname{dom}(p, s)$, and the right-hand side is an intersection of halfspaces. Similarly for $S_{3}$.

2. Otherwise, there exists an $i$ such that $\operatorname{dist}\left(x_{i}, x_{i+1}\right)<1 / 2$ for some $x_{i} \in$ $R_{i}$ and $x_{i+1} \in S_{i+1}$. By redefining $x_{i}$ and $x_{i+1}$ on the segment $x_{i} x_{i+1}$ if necessary, we have $x_{i} \in R_{i} \cap S_{i}$ and $x_{i+1} \in R_{i+1} \cap S_{i+1}$. Starting from this, we can inductively obtain a point $x_{j} \in R_{j} \cap S_{j}$ with $\operatorname{dist}\left(x_{j}, x_{j+1}\right)<1 / 2$ for each $j=i-1, i-2, \ldots, 0$; and a point $x_{j} \in R_{j} \cap S_{j}$ with $\operatorname{dist}\left(x_{j-1}, x_{j}\right)<1 / 2$ for each $j=i+2, i+3, \ldots, 4$. Thus $p$ and $q$ are connected by a path $x_{0} x_{1} x_{2} x_{3} x_{4}$ of length less than $4 \times 1 / 2$, a contradiction.

3 . We prove the first claim by induction on $i$ (the second claim is analogous). By part 2 and the induction hypothesis, $S_{i+1} \subseteq \mathbb{R}^{2} \backslash \mathrm{B}^{\circ}(p,(i+1) / 2)$. By this and $R_{i+1} \cup S_{i+1}=\mathbb{R}^{2}$, we have $R_{i+1} \supseteq \mathrm{B}^{\circ}(p,(i+1) / 2)$. Since $R_{i+1}$ is closed, $R_{i+1} \supseteq \mathrm{B}(p,(i+1) / 2)$.

4. Since $(0,0) \in S_{2}$ by part 3 , we have $R_{1}=\operatorname{dom}\left(\{p\}, S_{2}\right) \subseteq \operatorname{dom}(\{p\},(0,0))=$ $\mathbb{R} \times(-\infty,-1 / 2]$. Similarly for $S_{3}$.

5. Since $S_{3}$ is convex by part 1 , it is contained in the (closed) halfplane $H_{w}$ defined as the opposite side from $w$ across the perpendicular of $v w$ at $v$. Since $H_{w}$ contains $\mathrm{B}(q, 1 / 2)$ by part 3 , and the point $v$ on its boundary is in $\mathbb{R} \times[1 / 2, \infty)$ by part 4 , it must be the case that $0 \leq a^{\prime} \leq a$.

We will prove the other claim, $b^{\prime} \leq 0.82$. It suffices to show this for the case where $w$ is the upper-right-most point $w_{0}=(1.16,0)$. To see why, let $v_{0}=\left(a_{0}^{\prime}, b_{0}^{\prime}\right)$ be a closest point in $S_{3}$ to $w_{0}$, and define the halfplane $H_{w_{0}}$ in the same way as $H_{w}$ from $w_{0}$ and $v_{0}$. Since $H_{w_{0}}$ contains $w$ and $H_{w}$ contains $w_{0}$, they must coincide. So $v$ must be at the same relative position from $v_{0}$ as $w$ is from $w_{0}$, and thus must lie below, i.e., $b^{\prime} \leq b_{0}^{\prime}$.

Thus our goal now is to show $b_{0}^{\prime} \leq 0.82$. Recall that $H_{w_{0}}$ contains $\mathrm{B}(q, 1 / 2)$. The line tangent to the lower half of the ball $\mathrm{B}(q, 1 / 2)$ at the point with x-coordinate $x_{0}$ is described as

$$
y=\frac{x_{0}}{\sqrt{1 / 4-x_{0}^{2}}} x+\frac{\sqrt{1 / 4-x_{0}^{2}}-1 / 4}{\sqrt{1 / 4-x_{0}^{2}}} .
$$

Similarly, the line perpendicular to this tangent and passing through $w_{0}$ is

$$
y=-\frac{\sqrt{1 / 4-x_{0}^{2}}}{x_{0}} x+\frac{1.16 \sqrt{1 / 4-x_{0}^{2}}}{x_{0}} .
$$


These lines intersect at

$$
x=\frac{1.16 / 4+x_{0} / 4-1.16 x_{0}^{2}-x_{0} \sqrt{1 / 4-x_{0}^{2}}}{1 / 4+x_{0}^{2}} .
$$

Using substitution and a numerical calculation we get that the maximum $\mathrm{y}$-value achieved by this intersection point over the domain $x_{0} \in(0,1 / 2)$ is $0.819534 \ldots<0.82$ at $x_{0}=0.243934 \ldots$ This value bounds $b_{0}^{\prime}$ from above.

6. By parts 3 and $4, R_{2}=\operatorname{dom}\left(R_{1}, S_{3}\right) \subseteq \operatorname{dom}(\mathbb{R} \times(-\infty,-1 / 2], \mathrm{B}(q, 1 / 2))=$ $\operatorname{dom}(\mathbb{R} \times(-\infty,-1]),\{q\})=L_{4}^{+}$. Similarly for $S_{2}$.

In the rest of this paper, part $x$ of Lemma 6 is referred to as Lemma 6.x.

\subsection{Proof of Lemma 3: Uniqueness Near the Origin}

We now prove Lemma 3. Recall the statement:

Lemma 3. Let $\left(R_{i}, S_{i}\right)_{i}$ be a 4 -gradation. Then $R_{2}$ and $S_{2}$ agree with the trivial 4-gradation on $J^{\prime}:=J_{0.5774} \cap(\mathbb{R} \times[-4,4])$, i.e., $R_{2} \cap J^{\prime}=\hat{R}_{2} \cap J^{\prime}$ and $S_{2} \cap J^{\prime}=\hat{S}_{2} \cap J^{\prime}$.

Proof. We may assume that $\left(R_{i}, S_{i}\right)_{i}$ is the greatest 4-gradation in the sense of Lemma 2 (the same argument works for the least gradation by symmetry, and proves that the greatest and least gradations, and hence all gradations, coincide on $J^{\prime}$ ). This implies that $R_{i} \supseteq \hat{R}_{i}$ and $S_{i} \subseteq \hat{S}_{i}$ for each $i$. It suffices to prove that $R_{2} \cap J^{\prime} \subseteq \hat{R}_{2} \cap J^{\prime}$. Suppose otherwise, i.e., that $R_{2} \cap J^{\prime}$ contains a point above the x-axis. Then the set of $t>0$ with $L_{t}^{+} \supseteq R_{2} \cap J^{\prime}$ (see Lemma 6.6 for the definition of $L_{t}^{+}$) is bounded from above. This set is closed, and contains 4 by Lemma 6.6, so it has a maximum, which we call $t$ from now on. By the maximality of $t$, there is a point $w=(a, \varepsilon)$ in $\left(R_{2} \cap J^{\prime}\right) \backslash\{(0,0)\}$ on the boundary of $L_{t}^{+}$. This point $w$ is on the boundary of $R_{2}$. We assume without loss of generality that $a>0$. Calculation shows that the right uppermost point of $J^{\prime} \cap L_{4}^{+}$is $(1.15487 \ldots, 0.33343 \ldots)$, so $a<1.16$ and $\varepsilon<0.34$. Let $\hat{w}:=(a, 0)$.

Thus, we are looking at the point $w \in R_{2} \cap J^{\prime}$ that lies on the boundary of $L_{t}^{+}$. Below, we will split into two cases, and argue that in either case we can find another point $u \in R_{2} \cap J^{\prime}$ that does not belong to $L_{t}^{+}$, contradicting the way we defined $t$. Let $l:=\operatorname{dist}\left(w, R_{1}\right)=\operatorname{dist}\left(w, S_{3}\right)$ and $\hat{l}:=\operatorname{dist}\left(\hat{w}, \hat{R}_{1}\right)=\operatorname{dist}\left(\hat{w}, \hat{S}_{3}\right)$.

Case 1: $l \leq \hat{l}$. Define $v$ to be the closest point to $w$ in $R_{1}$, and let $\hat{v}:=v-(0, \varepsilon)$ (Fig. 3). Since $\operatorname{dist}(\hat{w}, \hat{v})=\operatorname{dist}(w, v)=l$, we have $\hat{v} \in \mathrm{B}(\hat{w}, l) \subseteq \mathrm{B}(\hat{w}, \hat{l}) \subseteq \hat{S}_{1}$.

Let $h:=\operatorname{dist}\left(v, S_{2}\right)$ and $\hat{h}:=\operatorname{dist}\left(\hat{v}, \hat{S}_{2}\right)$. Since $v \in \mathbb{R} \times[-0.82,0.82]$ by Lemma 6.5b, and $\varepsilon<0.34$, we have $h=\operatorname{dist}(v, p)>\operatorname{dist}(\hat{v}, p) \geq \hat{h}$.

Define $\hat{u}$ to be the closest point to $\hat{v}$ in $\hat{S}_{2}$ and let $u:=\hat{u}+(0, \varepsilon)=\left(a^{\prime}, \varepsilon\right)$. Thus, $u$ and $\hat{u}$ are vertically aligned with $\hat{v}$. Since $\operatorname{dist}(v, u)=\operatorname{dist}(\hat{v}, \hat{u})=\hat{h}<h$, the point $u$ belongs to $R_{2}$ and is different from $w$ (because $\operatorname{dist}(v, w) \geq h$ ). By Lemma 6.5a, it lies on the left of $w$, which was in $J^{\prime}$ and on the boundary of $L_{t}^{+}$. Hence, $u \in J^{\prime} \backslash L_{t}^{+}$, contradicting $R_{2} \cap J^{\prime} \subseteq L_{t}^{+}$. 


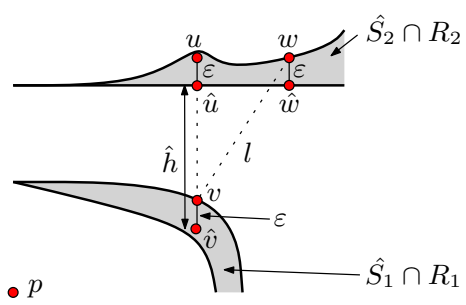

Fig. 3. Illustration of Case 1 from the proof of Lemma 3.

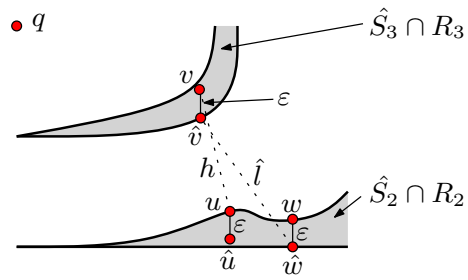

Fig. 4. Illustration of Case 2 from the proof of Lemma 3.

Case 2: $l \geq \hat{l}$. Define $\hat{v}$ to be the closest point to $\hat{w}$ in $\hat{S}_{3}$, and let $v:=\hat{v}+(0, \varepsilon)$ (Fig. 4). Since $\operatorname{dist}(w, v)=\operatorname{dist}(\hat{w}, \hat{v})=\hat{l}$, we have $v \in \mathrm{B}(w, \hat{l}) \subseteq \mathrm{B}(w, l) \subseteq R_{3}$.

Let $h:=\operatorname{dist}\left(v, R_{2}\right)$ and $\hat{h}:=\operatorname{dist}\left(\hat{v}, \hat{R}_{2}\right)$. Since $\hat{v} \in \mathbb{R} \times[-0.82,0.82]$ by Lemma $6.5 \mathrm{~b}$, and $\varepsilon<0.34$, we have $h \leq \operatorname{dist}(v, q)<\operatorname{dist}(\hat{v}, q)=\hat{h}$.

Define $u$ to be the closest point (or one of the closest points) to $v$ in $R_{2}$, and let $\hat{u}=u-(0, \varepsilon)$. Since $w \in J^{\prime} \cap R_{2} \subseteq J^{\prime} \cap L_{4}^{+}$by Lemma 6.6, the point $v$ is in the region $V$ in Fig. 5. Calculation shows that $V$ does not intersect $\operatorname{dom}\left(\mathbb{R}^{2} \backslash J^{\prime},\{q\}\right)$. In particular, $\operatorname{dist}\left(v, \mathbb{R}^{2} \backslash J^{\prime}\right)>\operatorname{dist}(v, q) \geq h=\operatorname{dist}(v, u)$. Hence $u \in J^{\prime}$.

Again, our goal is to prove $u \notin L_{t}^{+}$, thus contradicting $R_{2} \cap J^{\prime} \subseteq L_{t}^{+}$. Note that $u \in \mathrm{B}(v, \hat{l})$, because $u$ is a closest point to $v$ in $R_{2}$, and hence closer than $w$. Note also that $u \in \mathbb{R} \times(\varepsilon, \infty)$, because $\hat{u}$ lies in the interior of $\mathrm{B}(\hat{v}, \hat{h}) \subseteq \hat{S}_{2}$ by $\operatorname{dist}(\hat{v}, \hat{u})=\operatorname{dist}(v, u)=h<\hat{h}$. Thus, it suffices to prove that the three regions $L_{t}^{+}, \mathrm{B}(v, \hat{l})$ and $\mathbb{R} \times(\varepsilon, \infty)$ do not intersect. We argue as follows.

Notice that the boundaries of these three regions all pass through $w$. At this point $w$, the boundary of $\mathrm{B}(v, \hat{l})$ has slope $b$, where $b$ is the $\mathrm{x}$-coordinate of $\hat{v}$ and $v$, and the boundary of $L_{t}^{+}$has slope $2 a / t$, which is smaller than $b$ because $a=$ $\left(3+b^{2}\right) b / 2<2 b$ and $t \geq 4$. And also on the right of $w$, the slope of the boundary of $\mathrm{B}(v, \hat{l})$ continues to be greater than that of $L_{t}^{+}$, because the curvature of the former is greater than that of the latter by $\hat{l} \leq \operatorname{dist}\left((1.16,0), \hat{S}_{3}\right)=0.874 \ldots<1$. Hence, the boundaries of $\mathrm{B}(v, \hat{l})$ and $L_{t}^{+}$never meet on the right of $w$, so the intersection $\mathrm{B}(v, \hat{l}) \cap L_{t}^{+}$lies entirely below $w$, and thus misses $\mathbb{R} \times(\varepsilon, \infty)$. 


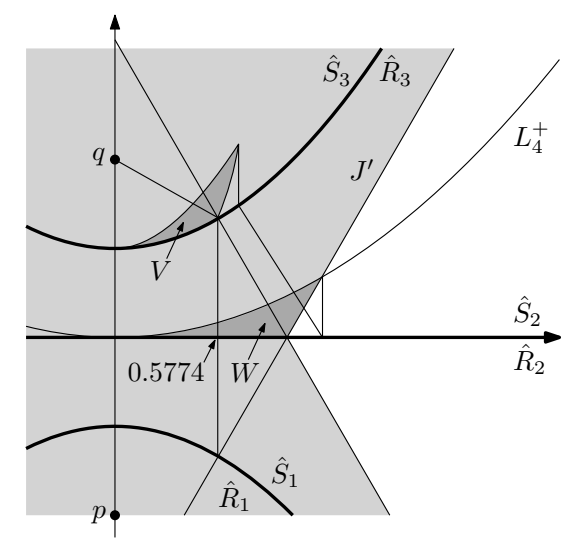

Fig. 5. The light-shaded region is $J^{\prime}$. Since the point $w$ is in the region $W=L_{4}^{+} \cap \hat{S}_{2} \cap J^{\prime}$, the point $v$ (obtained from $w$ by way of $\hat{w}$ and $\hat{v}$ as described in Case 2 of the proof of Lemma 3 ) is in the region $V$ (defined by suitable algebraic curves).

\subsection{Proof of Lemmas 4 and 5: Extending Uniqueness}

We define an additional closed region $K_{\beta}$. The boundaries of $K_{\beta}$ are line segments which are positioned at angles of $\pm \pi / 3$ with respect to the x-axis and intersect the boundary of $I_{\beta}$ on the x-axis, so the vertices bounding $K_{\beta}$ are $\left( \pm\left(3+\beta^{2}\right) \beta / 2,0\right)$ and $\left(0, \pm\left(3+\beta^{2}\right) \sqrt{3} \beta / 2\right)$. Note $I_{\beta} \subset K_{\beta}$ when $\beta>1 / \sqrt{3}$.

Recall Lemma 4:

Lemma 4. Let $\beta \geq 0.5774$. Let $\left(R_{i}, S_{i}\right)_{i}$ be a 4 -gradation. Suppose that $R_{2}$ and $S_{2}$ agree with the trivial 4-gradation on $J_{\beta}$, i.e., $R_{2} \cap J_{\beta}=\hat{R}_{2} \cap J_{\beta}$ and $S_{2} \cap J_{\beta}=\hat{S}_{2} \cap J_{\beta}$. Then $R_{1}, S_{1}, R_{3}, S_{3}$ agree with the trivial 4-gradation on a region $I_{\beta^{\prime}}$, for some $\beta^{\prime} \geq 1.00001 \beta$. Moreover, for $\beta=0.5774$, this holds with $J_{\beta}$ replaced by the $J^{\prime}$ defined in Lemma 3 .

Proof. Let $z \in K_{\beta}$. We demonstrate that $K_{\beta} \cap R_{1}=K_{\beta} \cap \hat{R}_{1}$; the argument is similar for $S_{1}, R_{3}, S_{3}$. The condition $\beta \geq 0.5774>1 / \sqrt{3}$ ensures that the lines delimiting $J_{\beta}$ have slope smaller (in absolute value) than $\sqrt{3}$, so that any point in $K_{\beta}$ is closer to $\hat{S}_{2} \cap J_{\beta}$ (which coincides with $S_{2} \cap J_{\beta}$ ) than to $\mathbb{R}^{2} \backslash J_{\beta}$. So the nearest point to $z$ in $S_{2}$ is in $J_{\beta}$. Hence

$$
\operatorname{dist}\left(z, S_{2}\right)=\operatorname{dist}\left(z, S_{2} \cap J_{\beta}\right)=\operatorname{dist}\left(z, \hat{S}_{2} \cap J_{\beta}\right)=\operatorname{dist}\left(z, \hat{S}_{2}\right) .
$$

So $z \in R_{1}$ if and only if $z \in \hat{R}_{1}$. Since $z$ was an arbitrary element of $K_{\beta}$, we have $K_{\beta} \cap R_{1}=K_{\beta} \cap \hat{R}_{1}$.

Define $\beta^{\prime}$ to be the absolute value of the x-coordinate of an intersection point of $R_{1}$ (or equivalently $\hat{R}_{1}$ ) with the boundary of $K_{\beta}$. Since $R_{1}$ is convex, it cannot contain a point in $I_{\beta^{\prime}} \backslash K_{\beta}$, and thus $I_{\beta^{\prime}} \cap R_{1}=I_{\beta^{\prime}} \cap \hat{R}_{1}$. 
It remains to prove $\beta^{\prime} \geq 1.00001 \beta$. To express $\beta^{\prime}$ in terms of $\beta$, it suffices to equate the expressions for $C_{1}$ and the boundary of $K_{\beta}$ and solve for the roots:

$$
\beta^{\prime}=-\sqrt{3}+\sqrt{2+\sqrt{3} \beta^{3}+3 \sqrt{3} \beta} .
$$

Finally, it may be shown that $\sigma^{\prime} \geq 1.00001 \sigma$ for $\sigma^{\prime}:=\sqrt{3} \beta^{\prime}$ and $\sigma:=\sqrt{3} \beta \geq$ $0.5774 \sqrt{3} \geq 1.00001^{8}$. Calculation shows that

$$
\sigma^{\prime}=-3+\sqrt{6+9 \sigma+\sigma^{3}} \geq-3+\left(3+\sigma^{9 / 8}\right)=\sigma^{1 / 8} \cdot \sigma \geq 1.00001 \sigma
$$

where the first inequality holds because $\left(6+9 \sigma+\sigma^{3}\right)-\left(3+\sigma^{9 / 8}\right)^{2}=3(\sigma-1)+$ $\sigma\left(\sigma^{10 / 8}+\sigma^{11 / 8}+\sigma^{12 / 8}+\sigma^{13 / 8}+\sigma^{14 / 8}+\sigma^{15 / 8}-6\right)\left(\sigma^{1 / 8}-1\right) \geq 0$.

For the final statement of the lemma (the "moreover" part), note that if $\beta=0.5774$, then $\left(3+\beta^{2}\right) \sqrt{3} \beta / 2<1.66$. Thus, $K_{0.5774} \subset \mathbb{R} \times[-1.66,1.66]$, and so points in $J_{0.5774} \backslash J^{\prime}$ are irrelevant.

Finally, we prove Lemma 5. Recall its statement:

Lemma 5. Let $\beta>0$. Let $\left(R_{i}, S_{i}\right)_{i}$ be a 4-gradation. Suppose that $R_{1}, S_{1}$, $R_{3}, S_{3}$ agree with the trivial 4-gradation on $I_{\beta}$, i.e., $R_{i} \cap I_{\beta}=\hat{R}_{i} \cap I_{\beta}$ and $S_{i} \cap I_{\beta}=\hat{S}_{i} \cap I_{\beta}$ for each $i=1,3$. Then $R_{2}, S_{2}$ agree with the trivial 4-gradation on $J_{\beta}$, i.e., $R_{2} \cap J_{\beta}=\hat{R}_{2} \cap J_{\beta}$ and $S_{2} \cap J_{\beta}=\hat{S}_{2} \cap J_{\beta}$.

Proof. Let $z \in J_{\beta}$. Because $R_{1}$ is convex (Lemma 6.1 ) and agrees with $\hat{R}_{1}$ on $I_{\beta}$, the nearest point to $z$ in $R_{1}$ is in $I_{\beta}$. Hence

$$
\operatorname{dist}\left(z, R_{1}\right)=\operatorname{dist}\left(z, R_{1} \cap I_{\beta}\right)=\operatorname{dist}\left(z, \hat{R}_{1} \cap I_{\beta}\right)=\operatorname{dist}\left(z, \hat{R}_{1}\right),
$$

and likewise

$$
\operatorname{dist}\left(z, S_{3}\right)=\operatorname{dist}\left(z, S_{3} \cap I_{\beta}\right)=\operatorname{dist}\left(z, \hat{S}_{3} \cap I_{\beta}\right)=\operatorname{dist}\left(z, \hat{S}_{3}\right) .
$$

So $z \in S_{2}$ if and only if $z \in \hat{S}_{2}$. Since $z$ was an arbitrary element of $J_{\beta}$, we have $J_{\beta} \cap S_{2}=J_{\beta} \cap \hat{S}_{2}$. Similarly for $R_{2}$.

This completes the proof of Theorem 1 .

\section{Conclusions}

We have shown that the 4-sector of two points is unique. Although we tried our best to simplify our proof, we still find it frustrating (and intriguing) that the uniqueness of such a seemingly basic object needed several pages to prove. As mentioned in the introduction, the main idea was to argue that if there are two $k$-sectors that differ at some point, there must be another point where they differ "more", in terms of some measure of the difference. But to implement this idea, we had to resort to calculation that relied heavily on the special setting of $k=4$ and $P, Q$ being points. It would be nice if this tedious calculation could be replaced by a conceptually simpler argument that works in more general settings. 


\section{References}

1. Asano, T., Kirkpatrick, D.: Distance trisector curves in regular convex distance metrics. In: Proc. 3rd International Symposium on Voronoi Diagrams in Science and Engineering. (2006) 8-17

2. Asano, T., Matoušek, J., Tokuyama, T.: Zone diagrams: Existence, uniqueness, and algorithmic challenge. SIAM Journal on Computing 37(4) (2007) 1182-1198

3. Asano, T., Matoušek, J., Tokuyama, T.: The distance trisector curve. Advances in Mathematics 212(1) (2007) 338-360

4. Asano, T., Tokuyama, T.: Drawing equally-spaced curves between two points. In: Proc. 14th Fall Workshop on Computational Geometry. (2004) 24-25

5. Chun, J., Okada, Y., Tokuyama, T.: Distance trisector of a segment and a point. Interdisciplinary Information Sciences 16(1) (2010) 119-125

6. Imai, K., Kawamura, A., Matoušek, J., Reem, D., Tokuyama, T.: Distance $k$ sectors exist. Computational Geometry 43(9) (2010) 713-720

7. Kawamura, A., Matoušek, J., Tokuyama, T.: Zone diagrams in Euclidean spaces and in other normed spaces. Mathematische Annalen 354(4) (2012) 1201-1221

8. Monterde, J., Ongay, F.: The distance trisector curve is transcendental. Geometriae Dedicata (in press).

9. Reem, D., Reich, S.: Zone and double zone diagrams in abstract spaces. Colloquium Mathematicum 115(1) (2009) 129-145

10. Reem, D.: On the computation of zone and double zone diagrams. arXiv:1208.3124 (2012) 